CASE OF THE MONTH

\section{Section Editors}

Robert C. Griggs, MD

Rochester, New York

Jerry R. Mendell, MD

Columbus, Ohio

Robert G. Miller, MD

San Francisco, California
ABSTRACT: A man with shoulder pain, wasting, and weakness had ipsilateral cranial nerve abnormalities. Electrodiagnostic studies supported a diagnosis of neuralgic amyotrophy, but we later demonstrated a spinal accessory mononeuropathy with ipsilateral hypoglossal weakness (ColletSicard syndrome). Magnetic resonance imaging demonstrated an inaccessible occipital condyle mass, and disseminated adenocarcinoma was subsequently diagnosed. Although cranial mononeuropathies can occur in neuralgic amyotrophy, this case illustrates the importance of identifying a focal lesion, and highlights the localizing value of electrodiagnosis. () 1997 John Wiley \& Sons, Inc. Muscle Nerve 20: 1173-1177, 1997

Key words: neuralgic amyotrophy; Collet-Sicard syndrome; spinal accessory neuropathy; adenocarcinoma; electromyography

\title{
COLLET-SICARD SYNDROME MIMICKING NEURALGIC AMYOTROPHY
}

\author{
WENDY L. LARSON, MD, AHMAD BEYDOUN, MD, \\ JAMES W. ALBERS, MD, PhD, and JOHN J. WALD, MD
}

Department of Neurology, University of Michigan Medical Center, 1500 E. Medical

Center Drive, Ann Arbor, Michigan 48109, USA

Accepted 31 M arch 1997

Neuralgic amyotrophy, idiopathic brachial plexopathy, or Parsonage-Turner syndrome is a clinical disorder characterized by the acute onset of shoulder and arm pain, followed by weakness and often wasting of involved muscles. ${ }^{18}$ Preceding trauma, infection, or immunization may be provoking factors. ${ }^{3}$ The upper trunk of the brachial plexus is commonly involved, as are the long thoracic and spinal accessory nerves. Electromyography is routinely used to localize the site and extent of abnormality, in addition to excluding patterns of nerve injury more likely due to a structural process.

Lower cranial neuropathies, while rare, may be due to structural lesions involving the skull base. Although idiopathic paralysis of the spinal accessory nerve occurs, it is frequently associated with subclinical involvement of neighboring muscles including the supraspinatus, deltoid, and serratus anterior. ${ }^{15}$ The pathophysiology of isolated spinal accessory mononeuropathy is thought to be similar to that of Parsonage-Turner syndrome. Clinical presentation involves severe pain frequently localized to the neck and top of the shoulder, weakness, and reduced

${ }^{\star}$ Correspondence to: Dr. John J. Wald

CCC 0148-639X/97/091173-05

(C) 1997 John Wiley \& Sons, Inc. shoulder range of motion. Paresthesias and numbness may occur, occasionally involving the entire arm. ${ }^{15}$

The following case illustrates the clinical dilemmas and electrodiagnostic challenges associated with evaluation of lower cranial neuropathies. Although the early presentation was suggestive of neuralgic amyotrophy, and initial electrodiagnostic studies supported that diagnosis, further evaluation revealed isolated spinal accessory nerve mononeuropathy with ipsilateral pharyngeal and hypoglossal abnormalities. We discuss this patient's evaluation and identify potential electrodiagnostic and clinical difficulties associated with the diagnosis of skull base masses involving the lower cranial nerves.

\section{CASE REPORT}

A 67-year-old man was referred to the University of Michigan Medical Center for evaluation of a 4-month history of severe unremitting right upper extremity pain associated with wasting of shoulder muscles. Symptoms began suddenly with sharp pain along the spinal edge of the right scapula, that radiated to involve the right shoulder and chest. A few weeks later, the patient noticed progressive loss of muscle mass around his neck and shoulder on the right side manifested by difficulty matching his shirt buttons when dressing. Thirteen weeks after the onset of symptoms, he reported difficulty swallowing. 
Past medical history was remarkable for rheumatoid arthritis and a 120 pack year history of tobacco use. Family history was remarkable for lung and pancreatic cancer in his father and younger brother, respectively.

The patient was first examined at the University of Michigan Medical Center 4 months following the onset of his symptoms. He was a tall cachectic man with stigmata of rheumatoid joint disease. There was a mild flaccid dysarthria, deviation of the tongue to the right, and a diminished gag reflex. The right sternocleidomastoid muscle was so atrophied that its belly could not be palpated (Fig. 1). There was atrophy and weakness of the right trapizius muscle associated with winging of the scapula with arm abduction, and apparent weakness of the right deltoid, right supraspinatus, and extensor neck muscles. Sensory examination was normal except for mild symmetric loss of position and vibratory sense in the lower extremities. Deep tendon reflexes were diffusely brisk without upper motor neuron signs.

Initial Evaluation. Electromyographic (EMG) data are presented in Tables 1 and 2 . The initial study revealed evidence of prominent partial denervation and reinnervation of the trapizius and supraspinatus muscles on the right, with less abnormal spontaneous activity in the right midcervical paraspinal muscles. Although clinically weak, the tongue was difficult to examine because of incomplete relaxation. There was a low trapezius motor evoked response amplitude on the right and incidental find- ings of mildly prolonged median and ulnar sensory responses and F-wave latencies. The electrodiagnostic findings were interpreted to be consistent with neuralgic amyotrophy involving the accessory nerve or with a structural or inflammatory lesion affecting the high cervical nerve roots and lower cranial nerves along with mild chronic ulnar and median mononeuropathies.

A brain magnetic resonance imaging (MRI) revealed a nonhomogeneous enhancing mass along the right anteroposterior basiocciput including the occipital condyle, jugular foramen, and hypoglossal foramen (Fig. 2). An MRI of the cervical spine was unremarkable. The lesion was not felt to be accessible to biopsy without significant risk. An evaluation for underlying malignancy or infection was unremarkable, including blood and urine cultures, examination of spinal fluid with cytology, and tuberculin skin test. Rheumatoid factor was elevated, but other serologies including complete blood count, electrolytes, serum chemistries, sedimentation rate, prostate specific antigen, carcinoembryonic antigen, and serum protein electrophoresis were unremarkable. Chest $X$ ray, computerized tomography of the chest, abdomen, and pelvis, and upper gastrointestinal series were normal with the exception of an irregularity of the duodenum. Endoscopy demonstrated chronic gastritis, and intestinal wall biopsies were negative for malignancy.

Clinical Course. Over the next several months, clinical examinations and serial imaging of the lower
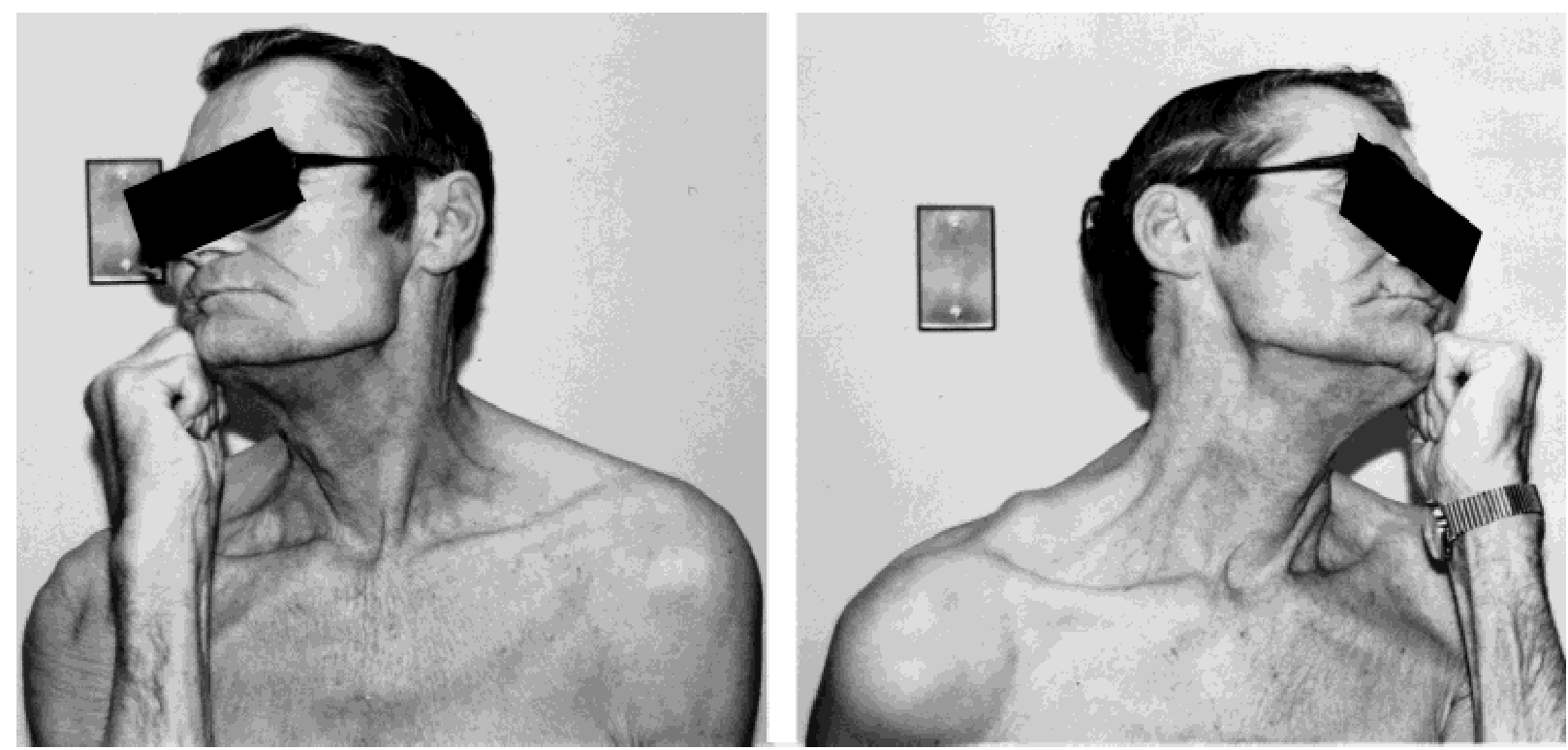

FIGURE 1. Photograph of the patient showing the right and left side of the neck. The severe atrophy of the right sternocleidomastoid muscle is apparent. 


\begin{tabular}{|c|c|c|c|c|}
\hline & \multicolumn{2}{|c|}{ Initial study (10/95) } & \multicolumn{2}{|c|}{ Follow-up study (2/96) } \\
\hline & $\begin{array}{c}\text { Fibrillation } \\
\text { potentials }\end{array}$ & $\begin{array}{l}\text { Motor } \\
\text { units }\end{array}$ & $\begin{array}{l}\text { Fibrillation } \\
\text { potentials }\end{array}$ & $\begin{array}{l}\text { Motor } \\
\text { units }\end{array}$ \\
\hline R supraspinatus & $\begin{array}{c}3+ \\
3+\end{array}$ & $\downarrow$ recruitment $\uparrow$ amplitude & Normal & Normal \\
\hline $\mathrm{R}$ pectoralis major & Normal & Normal & & \\
\hline $\mathrm{R}$ trapezius & $3+$ & None & $2+$ & None \\
\hline$L$ trapezius & Normal & Normal & Normal & Normal \\
\hline $\mathrm{R}$ deltoid & Normal & Normal & Normal & Normal \\
\hline $\mathrm{R}$ triceps & Normal & Normal & Normal & Normal \\
\hline R biceps & Normal & Normal & & \\
\hline R first dorsal interosseous & Normal & Slight, $\uparrow$ amplitude & & \\
\hline $\mathrm{R}$ abductor pollicis brevis & Normal & Slight, $\uparrow$ amplitude & & \\
\hline $\mathrm{R}$ extensor digitorum communis & & & Normal & Normal \\
\hline R extensor carpi ulnaris & & & Normal & Normal \\
\hline $\mathrm{R}$ rhomboids & & & Normal & Normal \\
\hline \multicolumn{5}{|l|}{$\mathrm{R}$ paraspinous muscles } \\
\hline High cervicals & $1+$ & $\uparrow$ polyphasia & & \\
\hline Low cervicals & Normal & $\uparrow$ polyphasia & Normal & Normal \\
\hline $\mathrm{R}_{\text {tongue }}$ * & Normal & & & \\
\hline
\end{tabular}

*Incomplete relaxation.

brain stem documented progression. During this time, the patient developed progressive dysarthria, dysphagia, and weight loss requiring placement of a gastrostomy tube to maintain adequate nutrition. Six months after the onset of his symptoms he developed sudden paralysis of extension of the right fourth and fifth fingers. Needle EMG evaluation demonstrated no abnormality of the extensor digi- torum communis or extensor carpi ulnaris. A palpable swelling in the region of the extensor tendons was evident over the dorsum of the hand, and the new weakness was due to a tendon tear. The remainder of the EMG examination (Tables 1 and 2) was normal except for evidence of denervation in the right trapezius muscle.

The spinal accessory mononeuropathy, progres-

\begin{tabular}{|c|c|c|c|c|c|}
\hline & \multicolumn{2}{|c|}{ Initial study (10/95) } & \multirow[b]{2}{*}{ Normal } & \multicolumn{2}{|c|}{ Follow-up study (2/96) } \\
\hline & Right & Left & & Right & Left \\
\hline \multicolumn{6}{|l|}{ Median (sensory) } \\
\hline Amplitude $(\mu \mathrm{V})$ & 10.9 & 16 & $>20$ & 8.7 & \\
\hline Distal latency (ms) & 4.0 & 3.4 & $<3.7$ & 4.1 & \\
\hline \multicolumn{6}{|l|}{ Median (motor) } \\
\hline Amplitude (mV) & 4.4 & & $>4$ & 3.6 & \\
\hline Distal latency (ms) & 4.2 & & $<4.4$ & 4.5 & \\
\hline Conduction velocity $(\mathrm{m} / \mathrm{s})$ & 51 & & $>49$ & 50 & \\
\hline F-wave latency (ms) & 33.5 & & $<32$ & 34.4 & \\
\hline \multicolumn{6}{|l|}{ Ulnar (sensory) } \\
\hline Amplitude $(\mu \mathrm{V})$ & 15.4 & & $>10$ & 14.3 & \\
\hline Distal latency (ms) & 4.0 & & $<3.5$ & 3.9 & \\
\hline \multicolumn{6}{|l|}{ Ulnar (motor) } \\
\hline Amplitude (mV) & 8.1 & & $>6$ & 5.8 & \\
\hline Distal latency (ms) & 3.1 & & $<3.5$ & 3.3 & \\
\hline Conduction velocity $(\mathrm{m} / \mathrm{s})$ & 65 & & $>49$ & 53 & \\
\hline F-wave latency (ms) & 35.3 & & $<33$ & 34.1 & \\
\hline \multicolumn{6}{|l|}{ Radial (sensory) } \\
\hline Amplitude $(\mu \mathrm{V})$ & 27.2 & & $>20$ & 13.5 & 28.7 \\
\hline Distal latency (ms) & 2.7 & & $<2.7$ & 2.6 & 2.3 \\
\hline \multicolumn{6}{|l|}{ Spinal accessory (trap) } \\
\hline Amplitude (mV) & 0.2 & & $>4.0$ & & \\
\hline Distal latency (ms) & 1.7 & & $<4.0$ & & \\
\hline
\end{tabular}



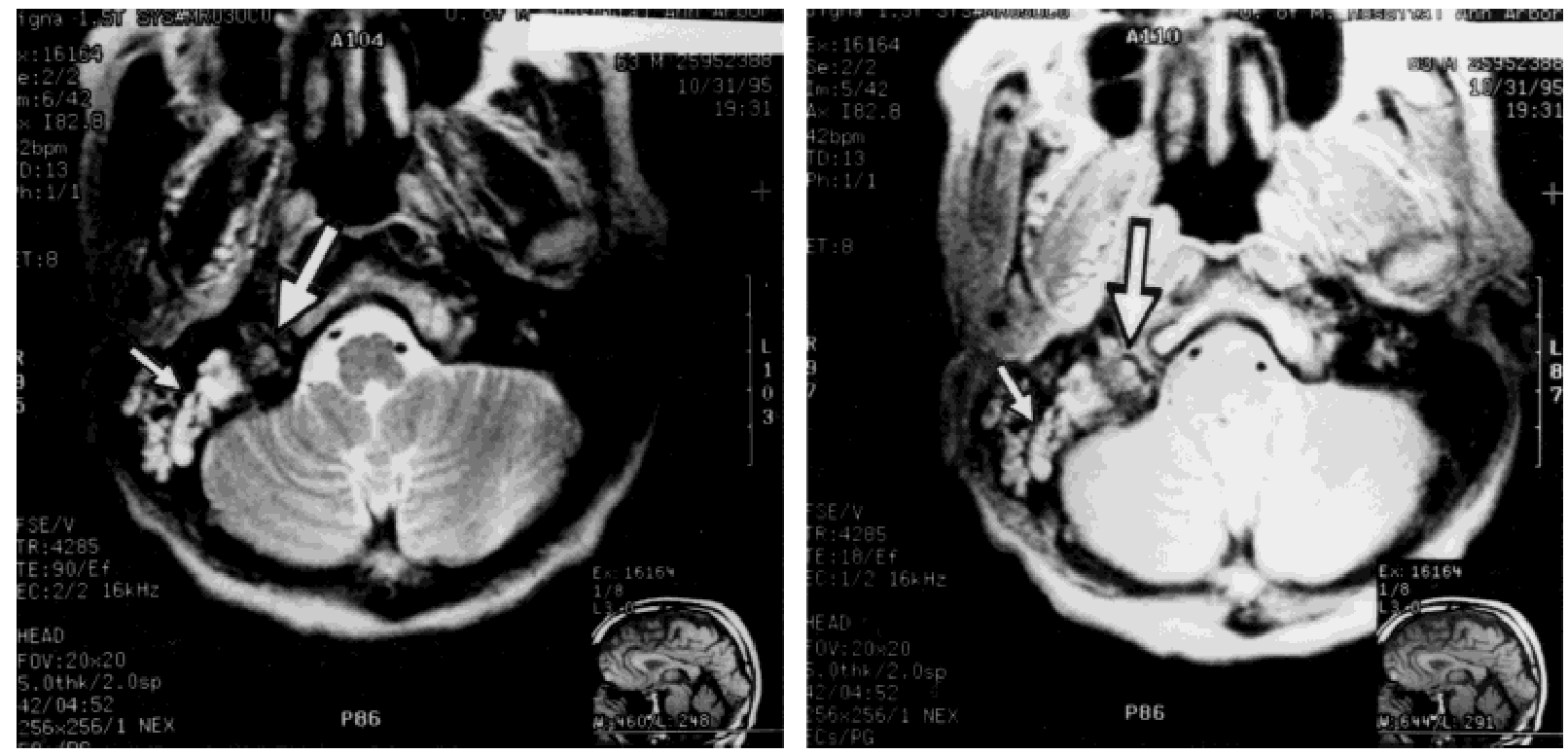

FIGURE 2. MRI scan of the brain stem showing a mass along the occipital condyle (large arrow), and fluid in the mastoid air cells (small arrow).

sive dysphagia, and ipsilateral hypoglossal weakness were interpreted as representing an infranuclear extramedullary lesion of cranial nerves 9-12 (ColletSicard syndrome). Additional evaluation for malignancy included a bone scan, which demonstrated abnormal uptake at the right skull base, right fourth rib, and left midfemur. Computed tomography (CT)-guided biopsy of the rib lesion revealed poorly differentiated adenocarcinoma. While receiving palliative radiation therapy and chemotherapy, the patient developed an aspiration pneumonia and died, 9 months after presentation.

\section{DISCUSSION}

This case illustrates a potential localizing pitfall in the electrodiagnostic evaluation of upper extremity pain and weakness, as well as the clinical challenge in diagnosing skull base masses. Although the early presentation of this patient with neuralgic pain followed by progressive weakness and atrophy of shoulder muscles was consistent with the diagnosis of neuralgic amyotrophy, the initial symptoms and signs were due to a spinal accessory mononeuropathy. The reported abnormalities in C5-C6 innervated muscles (supraspinatus and cervical paraspinals) on the initial EMG likely reflected inadvertent recording from the overlying, partially denervated trapezius muscle and erroneously led to a multifocal localization of the lesion.

Recent clinical and electrophysiological studies have shown that neuralgic amyotrophy is a diverse entity in which lesions are not restricted to the brachial plexus. $^{1,2,9,11,12}$ Mononeuropathy, mononeu- ropathy multiplex, and cranial nerve involvement can occur as part of this syndrome. In addition to the spinal accessory nerve, involvement of the glossopharyngeal, vagal, and hypoglossal nerves has been described, ${ }^{9,11}$ as has a case of neuralgic amyotrophy with simultaneous involvement of cranial nerves IXXII. ${ }^{9}$ The wide range of clinical manifestations in this disorder and the possibility of lower cranial nerve involvement can be diagnostically challenging. As our case demonstrates, when cranial nerve abnormalities are present, one needs to exclude other possibilities, especially lesions involving the base of the skull, before making the diagnosis of neuralgic amyotrophy.

The severity of muscle atrophy in the distribution of a single nerve, with almost complete loss of the sternocleidomastoid and trapezius muscles, in retrospect was atypical of an acute neuralgic amyotrophy. The coincidental development of a tendon tear in the affected extremity mimicking radial nerve or $\mathrm{C} 7$ root injury was diagnostically confusing until repeat electrodiagnostic studies were performed.

Combined abnormalities of cranial nerves IXXII without an associated H orner's syndrome (sympathetic chain spared) are commonly referred to as the Collet-Sicard syndrome. This syndrome has been described in idiopathic cranial polyneuropathy ${ }_{1}^{5}$ multiple myeloma, ${ }^{16}$ internal carotid artery dissection ${ }^{10}$ and coiling, ${ }^{14}$ closed head injury, ${ }^{13,17}$ Lyme disease, ${ }^{8}$ and in association with skull base tumors of primary and metastatic (prostate, lung, breast, and renal cell tumors) origin. ${ }^{7}$ In a review of skull base metastases, Greenberg et al. ${ }^{4}$ reported 43 patients, in 
whom the neurologic problem was the presenting symptom in only 2 patients. In $23 \%$ of their patients, the only evidence of metastasis was the skull base lesion. ${ }^{4}$ Adenocarcinoma of unknown primary origin comprises $3-15 \%$ of all cancers seen at referral centers. There is a 5-month median survival from first diagnostic procedure. Pancreas, lung, and gastrointestinal tract are the most common sites of tumor origin at autopsy. In 16\% of patients, however, autopsy does not reveal the site of origin. In their review, Le Chevalier et al. emphasized the importance of striking a balance between the value of a diagnosis versus the amount of patient discomfort, survival benefit, and cost. ${ }^{6}$

In summary, lower cranial mononeuropathies can present with symptoms suggesting neuralgic amyotrophy, particularly when the trapezius muscle is involved. Since the trapezius muscle helps stabilize the shoulder, its weakness may cause adjacent muscles to appear clinically weak. In addition, since movement of the shoulder is commonly painful, this leads to pseudoparesis and disuse atrophy in adjacent muscles. Because the trapezius overlays other shoulder muscles, needle electromyography may suggest, as it did in this patient, abnormal insertional activity in deeper muscles because of inadvertent placement of the examining electrode into the more superficial trapezius muscle. Once these difficulties are identified, however, the electrodiagnostic findings are helpful in localizing the site of the lesion.

\section{REFERENCES}

1. Arpa J, Cruz-Martinez A, Lacasa T: Recurrent amyotrophic neuralgia with an unusual clinical expression. Clinical and electrophysiological study. Neurologia 1991;6:65-67.

2. England JD, Sumner AJ: Neuralgic amyotrophy: an increasingly diverse entity. M uscle N erve 1987;10:60-68.
3. Gauthier JC, Bruyn GW: The serogenetic peripheral neuropathies, in Vinken PJ, Bruyn GW (eds): Handbook of Clinical Neurology. Amsterdam, North Holland Publishing Co, 1970, vol 8, pp 95-111.

4. Greenberg HS, Deck MD, Bikram B, Chu FCH, Posner JB: Metastasis to the base of the skull: clinical findings in 43 patients. N eurology 1981;31:530-537.

5. Junios JL, Beal MF: Idiopathic cranial polyneuropathy. Brain 1987;110:197-211.

6. Le Chevalier T, Cvitkovic E, Caille P, Harvey J, Contesso G, Spielmann M, Rouesse J: Early metastatic cancer of unknown primary origin at presentation. Arch Intern Med 1988;148: 2035-2039.

7. Mohanty SK, Barrios M, Fishbone H, Khatib R: Irreversible injury of cranial nerves 9 through 12 (Collet-Sicard syndrome). J Neurosurg 1973;38:86-88.

8. Pachner AR, Steere AC: The triad of neurologic manifestations of Lyme diseases: meningitis, cranial neuritis and radiculoneuritis. Neurology 1985;35:47-53.

9. Pierre PA, Laterre CE, Van Den Bergh PY: Neuralgic amyotrophy with involvement of cranial nerves IX, X, XI and XII. M uscle N erve 1990;13:704-707.

10. Ruiz J, Varona L, Martin-Gomez JI, Perez-Bas M, Mateos B, Zarranz JJ: Spontaneous internal carotid artery dissection as a cause of unilateral lower cranial nerve palsies. N eurologia 1995; 10:391-393.

11. Sanders EA, Van Den Neste V, Hoogenraad T: Brachial plexus neuritis and recurrent laryngeal nerve palsy. J Neurol 1988;235:323.

12. Serratrice G, Baudoin D, Pouget J, Blin O, Guieu R: Typical and atypical forms of neuralgic amyotrophy of the shoulder: 86 cases. Rev Neurol Paris 1992;148:47-50.

13. Sharma BS, Mahajan RK, Bhatia S, Khosla VK: Collet-Sicard syndrome after closed head injury. Clin N eurol Neurosurg 1994; 96:197-198.

14. Silvestrini M, Floris R, Tagliati M, Stanzione P, Simonetti G: Collet-Sicard syndrome caused by a coiling of the internal carotid artery. Riv Neurol 1991;61:135-136.

15. Sweeney PJ, Hanson MR: The cranial neuropathies, in Neurology in Clinical Practice. Boston, MA, ButterworthHeinemann, 1995, pp 1731-1732.

16. Tappin JA, Satchi G, Corless JA, Ashworth F: Multiple myeloma presenting as the Collet-Sicard syndrome. J N eurol $\mathrm{N}$ eurosurg Psychiatry 1996;60:14.

17. Wani MA, Tandon PN, Banerji AK, Bhatia R: Collet-Sicard syndrome resulting from closed head injury: case report. J Trauma 1991;31:1437-1439.

18. Wilbourn AJ: Brachial plexus disorders, in Dyck PJ, Thomas PK (eds): Peripheral Neuropathy. Philadelphia, W.B. Saunders, 1993, vol 2, pp 911-950. 